\title{
Check-list da Família Oxalidaceae no estado do Mato Grosso do Sul
}

\author{
Pedro Fiaschi
}

Universidade Federal de Santa Catarina, Centro de Ciências Biológicas, Campus Universitário, Departamento de Botânica, Trindade, CEP 88040-900, Florianópolis, Santa Catarina, Brasil. pedrofiaschi@gmail.com

Recebido em 27.XI.2014

Aceito em 16.IX.2015

DOI 10.21826/2446-8231201873s297

RESUMO - É apresentada uma listagem comentada das espécies nativas e exóticas invasoras de Oxalidaceae no estado do Mato Grosso do Sul. Há 17 espécies de Oxalis no Mato Grosso do Sul, das quais 14 são nativas e três exóticas invasoras. A lista do Mato Grosso do Sul é comparada com listas de outros estados brasileiros.

Palavras-chave: Cerrado, Chaco, flora, Mata Atlântica, Oxalis.

ABSTRACT - Checklist of the Oxalidaceae from Mato Grosso do Sul state, Brazil. A commented checklist of the native and exotic invasive species of Oxalidaceae from the state of Mato Grosso do Sul is presented. There are 17 species of Oxalis in Mato Grosso do Sul, of which 14 are native and three are exotic invasives. The checklist is compared with other Brazilian state lists.

Keywords: Atlantic Forest, Cerrado, Chaco, flora, Oxalis.

\section{INTRODUÇÃO}

A família Oxalidaceae possui cinco gêneros e aproximadamente 770 espécies, a maioria das quais (ca. 700) pertencente a Oxalis L., gênero que possui centros de diversidade na América do Sul (ca. 250 spp.) e no sul da África (ca. 210 spp.) (Oberlander et al. 2011). No Brasil as Oxalidaceae estão representadas por dois gêneros (Biophytum DC. e Oxalis L.) e aproximadamente 100 espécies nativas, além de duas espécies cultivadas de Averrhoa L. (Abreu \& Fiaschi 2015). As espécies brasileiras de Biophytum estão concentradas na Amazônia Ocidental, ao passo que Oxalis ocorre em todo o país, tanto em áreas tropicais, onde são mais comuns espécies com folhas pinadas trifolioladas (Lourteig 1994), quanto subtropicais, onde abundam espécies com folhas digitadas trifolioladas (Lourteig 2000).

\section{Principais acervos e lacunas de conhecimento}

Os principais acervos com amostras de Oxalidaceae do Mato Grosso do Sul são os herbários CGMS (Universidade Federal do Mato Grosso do Sul, Campo Grande) e MBM (Museu Botânico Municipal, Curitiba).

Não há até o momento grupos de pesquisa dedicados às Oxalidaceae no Brasil. Estudos colaborativos sobre as Oxalis encontram-se em andamento entre pesquisadores da África do Sul, Alemanha, Argentina, Brasil, Estados Unidos e Uruguai. Algumas contribuições relevantes ao conhecimento da família no Brasil são apresentadas na bibliografia (abaixo).

As relações filogenéticas entre espécies de Oxalis subgênero Thamnoxys, que inclui a maioria das espécies de Oxalis no Mato Grosso do Sul, ainda não foram elucidadas. Algumas das seções adotadas por Lourteig (1994, 2000) parecem não ser monofiléticas (Gardner, comunicação pessoal) e a delimitação de algumas espécies precisa ser revista, de preferência por meio de testes de monofiletismo. Estudos futuros das Oxalidaceae do Mato Grosso do Sul se beneficiariam da intensificação de coletas em áreas do Domínio da Mata Atlântica e em áreas remanescentes do Chaco, na porção Sudoeste do estado. A inclusão de exemplares do Mato Grosso do Sul em estudos filogenéticos ampliará a amostragem para testar o monofiletismo de Oxalis sect. Thamnoxys (Endl.) Reiche emend. Lourteig, $O$. sect. Robustae (Progel) Lourteig e O. sect. Psoraleoideae Lourteig, e permitirá posteriores revisões taxonômicas e a circunscrição de espécies em grupos delimitados filogeneticamente.

\section{MATERIAL E MÉTODOS}

Foram consultados para a elaboração desta lista os herbários CGMS, MBM e SPF (acrônimos segundo Thiers 2012), além da lista de espécies de Oxalidaceae divulgada pelo projeto Lista de espécies da Flora do Brasil (Abreu \& Fiaschi 2012). 


\section{RESULTADOS E DISCUSSÃO}

A lista das espécies de Oxalidaceae nativas ou exóticas invasoras no estado do Mato Grosso do Sul é apresentada abaixo.

Oxalidaceae R.Br.

Oxalis barrelieri $\mathrm{L}$.

Erva ereta com folhas pinadas trifolioladas, lâmina glabrescente ou com tricomas esparsos na face abaxial e flores rosadas com a fauce amarela. A espécie possui distribuição ampla na América Tropical.

Material examinado: G. Hatschbach 21785 (MBM), G. Hatschbach 22040 (MBM).

Oxalis conorrhiza (Feuillée) Jacq.

Erva ereta com folhas digitadas trifolioladas, densamente agrupadas, inflorescências unifloras e flores amarelas. Distribuição ampla no sul e sudeste do Brasil e países vizinhos.

Material examinado: G. Hatschbach 48421 (MBM).

\section{Oxalis corniculata $\mathrm{L}$.}

Erva prostrada com folhas digitadas trifolioladas, inflorescências (1)2-3-floras e flores amarelas. Espécie exótica invasora originária da Europa, com distribuição cosmopolita. Material examinado: A. Pott 8400 (CGMS), A. Pott 8471 (CGMS), V.J. Pott 7432 (CGMS).

\section{Oxalis cratensis Oliv.}

Erva ereta, glabra ou glabrescente, com folhas pinadas trifolioladas e flores rosadas com a fauce amarela. Ocorre no Brasil, Paraguai e Bolívia, geralmente na borda de florestas. Representada no estado principalmente por $O$. cratensis var. paraguayensis (Knuth) Lourteig.

Material examinado: G. Hatschbach 29449 (MBM), G. Hatschbach 60820 (MBM).

\section{Oxalis debilis Kunth}

Erva bulbosa com folhas digitadas trifolioladas, inflorescências umbeliformes 8-11-floras e flores rosadas. Espécie exótica invasora originária dos Andes tropicais, com distribuição cosmopolita devido à introdução como planta ornamental.

Material examinado: A. Pott 8470 (CGMS).

Oxalis cytisoides Mart. ex Zucc.

Subarbusto ereto, pubescente, com folhas pinadas trifolioladas e flores rosadas com a fauce amarela. Ocorre no Brasil e no Nordeste da Argentina (Misiones), geralmente em bordas de florestas.

Material examinado: G. Hatschbach 73257 (MBM), G. Hatschbach 74713 (MBM).

\section{Oxalis erosa Knuth}

Arbusto curto-pubescente, com folhas pinadas trifolioladas, inflorescências umbeliformes 7-11-floras, flores amarelas ou rosadas e frutos com carpelos unisseminados. Ocorre na Bolívia, Paraguai, Argentina e no Sudoeste do Mato Grosso do Sul, na pequena extensão do domínio do Chaco no Brasil.

Material examinado: G. Hatschbach 49231 (MBM), G. Hatschbach 77259 (MBM), G. Hatschbach 76235 (MBM), G. Hatschbach 76973 (MBM), J.M. Silva 5319 (MBM)

\section{Oxalis frutescens L. ssp. frutescens}

Erva ou arbusto ereto com folhas pinadas trifolioladas, fasciculadas em braquiblastos, flores amarelas e frutos com carpelos 1-4-seminados. A espécie possui distribuição ampla na América Tropical, com ocorrência no Pantanal sul-matogrossense.

Material examinado:A. Pott 4138 (MBM), A. Pott 5302 (MBM), G. Hatschbach 30448 (MBM), G. Hatschbach 60798 (MBM).

\section{Oxalis glaucescens Norlind}

Erva prostrada com folhas pinadas trifolioladas, folíolo terminal com ápice retuso ou emarginado, flores amarelas e frutos com o cálice aberto, assemelhando-se a uma "estrela". A espécie é comum nas caatingas do Nordeste do Brasil, e possui distribuição disjunta no Mato Grosso do Sul, onde foi coletada apenas em Corumbá.

Material examinado: V.J. Pott 70 (CGMS)

\section{Oxalis grisea A.St.-Hil. \& Naudin}

Arbusto ou subarbusto pubescente, com folhas pinadas trifolioladas, folíolo terminal geralmente obovado, inflorescências umbeliformes, flores amarelas e frutos com carpelos 2-3-seminados. Ocorre em cerrados na Bolívia, Brasil e Paraguai. Oxalis physocalyx Zucc. ex Prog. não é aqui reconhecida como distinta de $O$. grisea, embora Lourteig (1994) tenha indicado que ambas são encontradas no estado e podem ser distintas pelo indumento dos ramos e dos carpelos. Uma das coletas atribuídas a $O$. physocalyx por esta autora é listada abaixo seguida por um asterisco. Material examinado: A. Krapovickas 34407 (MBM), A. Krapovickas 34481 (MBM), G. Hatschbach 23601 (MBM), G. Hatschbach 23645 (MBM), G. Hatschbach 23557 (MBM), G. Hatschbach G. Hatschbach 31975 (MBM), G. Hatschbach 49133 (MBM), G. Hatschbach $58666(\mathrm{MBM})^{*}$, G. Hatschbach 63416 (MBM), G. Hatschbach 73161 (MBM), G. Hatschbach 74014 (MBM), G. Hatschbach 74307 (MBM), G. Hatschbach 74941 (MBM), G. Hatschbach 76978 (MBM), G. Hatschbach 77034 (MBM), G. Hatschbach 77133 (MBM), G.J. Shepherd 4134 (MBM), H.F. Leitão Filho 2107 (MBM), J.M. Silva 5304 (MBM), P.E. Gibbs 5437 (MBM), T.M. Pedersen 12174 (MBM).

\section{Oxalis hyalotricha Lourteig ssp. hyalotricha}

Subarbusto com tricomas alongados pluricelulares, folhas pinadas trifolioladas, flores amarelas e frutos com lóculos 4-6-seminados. Ocorre apenas na Bacia do Paraná, no Paraguai, Brasil e Argentina. 
Material examinado: L.R.H. Bicudo et al. 217 (CGMS, SPF).

\section{Oxalis psoraleoides Kunth ssp. insipida Lourteig}

Arbusto pubescente, com folhas pinadas trifolioladas, folíolo terminal elíptico a lanceolado, tomentoso na face abaxial, inflorescências umbeliformes, flores amarelas e frutos com carpelos unisseminados. Ocorre no Brasil (principalmente na Caatinga), Bolívia e no norte da Argentina e do Paraguai. Material examinado: G.A. Damasceno Júnior 2889 (CGMS).

\section{Oxalis renifolia Knuth}

Subarbusto com folhas unifolioladas, lâmina reniforme, flores amarelas, rosadas ou brancas. Espécie pouco comum, coletada apenas em Bela Vista. Ocorre no Chaco.

Material examinado: G. Hatschbach 76492 (MBM).

Oxalis rhombeo-ovata A.St.-Hil.

Subarbusto com folhas pinadas trifolioladas, folíolo terminal rombeo-ovado com margem ciliada, flores amarelas e cápsulas com lóculos unisseminados. A espécie é comum em florestas estacionais do sudeste e sul do Brasil, Paraguai e nordeste da Argentina (Misiones).

Material examinado: A.L.B. Sartori 438 (CGMS), G.A. Damasceno Júnior et al. 1640 (CGMS), V.J. Pott 3836 (CGMS)

\section{Oxalis sellowii Spreng.}

Erva ou subarbusto com folhas pinadas trifolioladas, folíolos geralmente lineares e pubescentes (às vezes glabros), flores amarelas, brancas ou rosadas e cápsulas com lóculos 2-3-seminados. Ocorre no Brasil, Paraguai e Argentina. Domínio da Mata Atlântica.

Material examinado: G. Hatschbach 25035 (MBM), G. Hatschbach 25162 (MBM), G. Hatschbach 45891 (MBM), G. Hatschbach 48419 (MBM), G. Hatschbach 48431 (MBM), G. Hatschbach 76876 (MBM).

\section{Oxalis sepium A.St.-Hil.}

Erva ou subarbusto com folhas pinadas trifolioladas, folíolos geralmente elípticos e glabrescentes, flores amarelas e cápsulas com lóculos 1-3-seminados. Ocorre no leste do Brasil, principalmente no domínio da Mata Atlântica. O único material examinado foi identificado por Lourteig (1994) como pertencente a O. hedysarifolia Raddi, espécie sem ocorrência confirmada no estado.

Material examinado: G. Hatschbach 25070 (MBM).

\section{Oxalis triangularis A.St.-Hil.}

Erva com rizoma coberto com escamas constituídas pelas bases foliares, folhas digitadas trifolioladas, inflorescências umbeliformes e flores rosadas ou quase alvas. Espécie nativa da América do Sul, com ocorrência no Brasil, Argentina, Paraguai e Bolívia.

Material examinado: A. Guglieri 1706 (CGMS), A. Pott 8357 (CGMS), A. Pott 10487 (CGMS), G. Hatschbach
63425 (MBM), T.H. Stefanello 220 (CGMS).

\section{Ocorrências não confirmadas}

\section{Oxalis hedysarifolia Raddi}

Erva ou subarbusto ereto, hirsuto, folhas pinadas trifolioladas, lâmina pubescente na face abaxial e flores amarelas. Ocorre na Bolívia, Brasil e Argentina, geralmente em bordas de florestas. Não foi possível confirmar a ocorrência desta espécie no estado. Lourteig (1994) citou as seguintes amostras da espécie no Mato Grosso do Sul: Brilhante, G. Hatschbach 25070 (MBM); Aquidauana, Piraputunga, G. Hatschbach 29449b (MBM).

\section{Oxalis latifolia Kunth}

Erva bulbosa com folhas digitadas trifolioladas, inflorescências umbeliformes 8-11-floras e flores rosadas. Espécie exótica invasora originária dos Andes tropicais, com distribuição cosmopolita devido à introdução como planta ornamental. Ocorrência provável no estado, porém não confirmada pela falta de amostras.

\section{Oxalis nigrescens A.St.-Hil.}

Erva ou subarbusto ereto, glabrescente, com folhas pinadas trifolioladas, folíolos elípticos ou lineares e flores geralmente amarelas. Ocorre em campos e cerrados no leste do Brasil, sendo especialmente comum em Minas Gerais e Goiás. Não foi possível confirmar a ocorrência desta espécie no estado. Abreu (2011) citou as seguintes amostras da espécie no Mato Grosso do Sul: Bodoquena, Rod. Bodoquena a Morraria do Sul, G. Hatschbach 73992 (MBM); Bonito, Assentamento Guaicurus, J.M. Silva 4921 (MBM); Caracol, Rio Perdido, J.M. Silva 4841 (MBM); Porto Murtinho, Faz. Nossa Senhora do Perpétuo Socorro, G. Hatschbach 74027 (MBM).

\section{Comentários}

As Oxalidaceae nativas ou exóticas invasoras do estado do Mato Grosso do Sul somam 17 espécies, todas pertencentes a Oxalis L. A maioria das espécies de Oxalis do estado pertence a Oxalis subg. Thamnoxys (Endl.) Reiche emed. Lourteig, cuja maior diversidade está nos campos e cerrados do Brasil central e na Mata Atlântica (Lourteig 1994). Espécies deste subgênero são geralmente arbustivas ou subarbustivas com folhas pinadas trifolioladas (às vezes unifolioladas). As demais espécies encontradas no estado pertencem a Oxalis subg. Oxalis, sendo a maioria destas exóticas naturalizadas em áreas antropizadas.

A lista de espécies nativas e naturalizadas de Oxalis do Mato Grosso do Sul é menor que a do estado de São Paulo, com 23 espécies (Fiaschi \& Conceição 2005), porém maior que a do Distrito Federal, com apenas 9 espécies registradas (Fiaschi, 2006). A riqueza do gênero no Mato Grosso do Sul é, entretanto, consideravelmente menor que a dos estados da região Sul do Brasil, como Rio Grande do Sul (27 spp., Grigoletto et al. 2014), Santa Catarina (29 spp., Lourteig 1983) e Paraná (32 spp., Fiaschi 2014). Nesses estados, a maior diversidade da família deve-se essencialmente à maior 
Quadro 1. Ocorrência dos táxons de Oxalidaceae por macrorregião no Mato Grosso do Sul.

\begin{tabular}{|c|c|c|c|c|}
\hline Táxon & Cerrado & Chaco & Mata Atlântica & Pantanal \\
\hline Oxalis barrelieri $\mathrm{L}$. & & & $\mathrm{X}$ & $\mathrm{X}$ \\
\hline O. conorrhiza (Feuillée) Jacq. & & & $\mathrm{X}$ & \\
\hline O. corniculata $\mathrm{L}$. & $\mathrm{X}$ & $\mathrm{X}$ & $\mathrm{X}$ & $\mathrm{X}$ \\
\hline \multicolumn{5}{|l|}{ O. cratensis Oliv. } \\
\hline O. cytisoides Mart. ex Zucc. & & & $\mathrm{X}$ & \\
\hline O. debilis Kunth & $\mathrm{X}$ & $\mathrm{X}$ & $\mathrm{X}$ & $\mathrm{X}$ \\
\hline O. erosa Knuth & & $\mathrm{X}$ & & \\
\hline O. frutescens ssp. frutescens $\mathrm{L}$. & & & & $\mathrm{X}$ \\
\hline O. glaucescens Norlind & & & & $X$ \\
\hline O. grisea A.St.-Hil. \& Naudin & $\mathrm{X}$ & $\mathrm{X}$ & & \\
\hline O. hyalotricha ssp. hyalotricha Lourteig & & & $\mathrm{X}$ & \\
\hline O. psoraleoides Kunth ssp. insipida Lourteig & & & & $X$ \\
\hline O. renifolia Knuth & & $X$ & & \\
\hline O. rhombeo-ovata A.St.-Hil. & & & $\mathrm{X}$ & \\
\hline O. sellowii Spreng. & & & $\mathrm{X}$ & \\
\hline \multicolumn{5}{|l|}{ O. sepium A.St.-Hil. } \\
\hline O. triangularis A.St.-Hil. & $\mathrm{X}$ & $\mathrm{X}$ & $\mathrm{X}$ & $\mathrm{X}$ \\
\hline
\end{tabular}

extensão e diversidade fisionômica do domínio da Mata Atlântica no sul e sudeste do Brasil do que no Centro-Oeste. Além disso, nos estados do sul do país existe uma riqueza maior de espécies de Oxalis subg. Oxalis, que totalizam mais de $96 \%$ das espécies de Oxalis no Rio Grande do Sul, ca. $86 \%$ das espécies no Paraná e em Santa Catarina, e apenas ca. $23.5 \%$ da riqueza de Oxalis no Mato Grosso do Sul.

A presença de uma pequena extensão do domínio do Chaco no Mato Grosso do Sul contribui de maneira significativa para a diversidade de Oxalis no estado, uma vez que duas das 17 espécies listadas ocorrem predominantemente em áreas chaquenhas: Oxalis erosa Knuth e Oxalis renifolia Knuth.

\section{AGRADECIMENTOS}

Agradeço à Dra. Maria Ana Farinaccio, da Universidade Federal do Mato Grosso do Sul, pelo convite para participar da compilação do Checklist, à Dra. Lezilda Torgan, pelo auxílio editorial, e aos curadores dos herbários visitados.

\section{REFERÊNCIAS}

Abreu, M.C. 2011. Sistemática de Oxalis L. sect. Thamnoxys (Endl.) Progel (Oxalidaceae) no Brasil. Tese 137 f., Universidade Federal Rural de Pernambuco, Recife.
Abreu, M.C. \& Fiaschi, P. 2015. Oxalidaceae. In Lista de Espécies da Flora do Brasil. Jardim Botânico do Rio de Janeiro. Disponível em: $<\mathrm{http}$ //floradobrasil.jbrj.gov.br/jabot/floradobrasil/FB181>. Acesso em: 15 Set. 2015.

Fiaschi, P. 2006. Oxalidaceae. In Flora do Distrito Federal, Brasil (T.B. Cavalcanti, org.), v. 5, p. 93-105.

Fiaschi, P. 2014. Oxalidaceae. In Plantas vasculares do Paraná (M. Kaehler, R. Goldenberg, P.H.L. Evangelista, O.S. Ribas, A.O.S. Vieira \& G.G. Hatschbach, eds.). Universidade Federal do Paraná, Curitiba, p. 156-157.

Fiaschi, P. \& Conceição, A.A. 2005. Oxalidaceae. In Flora Fanerogâmica do Estado de São Paulo (M.G.L. Wanderley, G.J. Shepherd, T.S. Melhem \& A.M. Giuletti, eds.) v. 4, p. 301-315.

Grigoletto, D., Bertuzzi, T., Eisinger, S.M., Canto-Dorow, T.S. \& Colusso, C.S. 2014. O gênero Oxalis L. (Oxalidaceae) no Rio Grande do Sul, Brasil. Ciência e Natura, Santa Maria 36(ed. especial II): 594-612.

Lourteig, A. 1983. Oxalidáceas. In Flora Ilustrada Catarinense (R. Reitz, ed.). Herbário Barbosa Rodrigues, Itajaí, Santa Catarina, Brasil. . 1994. Oxalis L. Subgénero Thamnoxys (Endl.) Reiche emend. Lourteig. Bradea 7(1): 1-197.

. 2000. Oxalis L. Subgêneros Monoxalis (Small) Lourt., Oxalis y Trifidus Lourt. Bradea 7(2): 201-629.

Oberlander, K.C., Dreyer, L.L. \& Bellstedt, D.U. 2011. Molecular phylogenetics and origins of southern African Oxalis. Taxon 60(6): 1667-1677.

Thiers, B. 2012. Index Herbariorum: A global directory of public herbaria and associated staff. New York Botanical Garden's Virtual Herbarium. Disponível em: http://sweetgum.nybg.org/ih/. Acessado em 29.08.2012. 